\title{
Doping-Induced Type-II to Type-I Transition and Interband Optical Gain in InAs/AlSb Quantum Wells
}

\author{
K. I. Kolokolov and C. Z. Ning* \\ Center for Nanotechnology, NASA Ames Research Center, \\ MS N229-1, Moffett Field, CA 94035-1000 \\ (Dated: )
}

\begin{abstract}
We show that proper doping of the barrier regions can convert the well-known type-II InAs/AlSb QWs to type I, producing strong interband transitions comparable to regular type-I QWs. The interband gain for TM mode is as high as $40001 / \mathrm{cm}$, thus providing an important alternative material system in the mid-infrared wavelength range. We also study the TE and TM gain as functions of doping level and intrinsic electron-hole density.
\end{abstract}

* corresponding author: email: cning@mail.arc.nasa.gov 
It is well-known that InAs/AlSb QWs have type-II bandedge lineups where spatial separation of electrons and holes leads to week or vanishing interband dipole matrix elements, except for very narrow InAs layers of a few monolayers. This fact precludes InAs/AlSb from being used as an interband gain material. This is particularly an unfortunate situation since the bandgap of InAs is in the mid-wave infrared wavelength (MWIR) region, where applications are abundant, yet light sources are scarce and still to achieve mature status. While photoluminescence has been measured for InAs QWs[1-4], yet there has not been a device application based on interband transitions. Most of recent papers on this material system focus on intersubband transitions[5-7] and the possible device applications in the MWIR and THz wavelength ranges.

In this letter, we show that proper doping in the InAs/AlSb heterostructure converts an InAs layer from a barrier to a well for holes, thus converting a type-II heterostructure into a type-I due to the associated band bending. We show that the resultant dipole matrix elements and the interband gain are comparable to those of typical type-I structure. Both dipole moments and optical gain increase significantly with doping level. TM mode optical gain can be as high as $40001 / \mathrm{cm}$. We believe that such doped InAs/AlSb quantum well may provide an interesting alternative to the materials system for MWIR generation.

The model structure we consider is an InAs layer of $10 \mathrm{~nm}$ sandwiched between two AlSb layers of $21 \mathrm{~nm}$ each. A fraction (10 nm in thickness) of the AlSb layers on both sides of the well is doped uniformly with a total $2 \mathrm{D}$ density in the range of $1 \times$ to $5 \times 10^{12} \mathrm{~cm}^{-2}$. Between the InAs and the doped layer there is an undoped spacer layer of $6 \mathrm{~nm}$ on both sides of the QW. We start by performing a self-consistent band structure calculation based on the 8 band $\vec{k} \cdot \vec{p}$ model with strain effects treated using the standard Pikus-Bir model, coupled to the Poisson equation. In this paper we assume that AlSb layers are unstrained as is often the case in such samples and therefore the InAs QW is subject to tensile strain. Such a calculation is more or less standard [8] and the equations will not be repeated here. The only difference is that we use the approach of Ref. [9] to avoid the spurious states. Typical values of parameters are chosen from Ref. [10]. We mention in particular the valence band offset between InAs and $\mathrm{AlSb}$ chosen to be $180 \mathrm{meV}$, which is the upper limit in the range of uncertainty. The required doping densities will be reduced if smaller valence band offset parameter is used. In addition to the doping density, we also assume that electron hole pairs 
(called e-h density) are present which could be optically generated or injected electrically. In the following, we vary the values of the doping density and the e-h density for a given study.

Fig. 1 shows bulk band edge profiles for conduction, heavy, and light hole bands, as well as the corresponding wave functions, calculated self-consistently. Without doping this is a type-II structure for low e-h density with vanishing spatial overlap between electrons and holes. This is still evident from Fig.1(a) where doping density is relatively low. Band bending induced by high density of e-h pairs without additional doping can give rise to finite, but small optical gain due to the pulling of holes toward the well-barrier interface[3, 11]. When the structure is doped, strong band bending occurs near the interface regions. Due to the tensile strain of the InAs layer and the associated bandedge splitting of heavy-light hole bands, the barrier for light holes is smaller than for heavy holes. As a result, the two triangle QWs formed by doping are more strongly coupled for light holes, leading to a finite probability of light holes in the InAs layer (see dash-dotted line in Fig.1a). The wave function for heavy holes is still practically zero at the center of InAs layer. The ground state at this doping density is heavy hole band. With a further increase of doping density to $3 \times 10^{12} \mathrm{~cm}^{-2}$ as shown in Fig.1(b), strong band bending pushes the InAs valence band edges upwards further energetically and above the original AlSb valence bandedge as shown in Fig.1(b). The InAs layer is no longer a global barrier for both heavy and light holes. In fact, the first light hole subband, which becomes now the ground state, is energetically lower than the central portion of the bulk light hole bandedge in InAs layer. For light holes in the ground state, the structure becomes similar to three coupled QWs: two triangle wells at the interfaces and one parabolic well in the center of the InAs layer. This is clearly seen in the wave function of the light hole band (dashed curve in Fig.1b), which shows three maximums indicating the presence of the three coupled wells. As a result, we see that light hole wavefunction is strongly concentrated in the InAs layer where electrons are confined. Thus the structure now becomes practically a type-I QW for light holes. Since the first heavy hole band is still energetically higher than its bandedge profile (thick dashed line) in the InAs region, the structure is still like coupled double wells for heavy holes. Heavy holes are still predominately in the interface area with very small amplitude in the center of InAs layer. It is interesting and important to note that the ground state changes from heavy hole 
band at low doping density (Fig.1(a)) to light hole band at high doping density (Fig.1(b)). At the low doping density, the heavy hole band is energetically higher because of the heavy mass. At the high doping density, however, the strong band bending leads to an effective, much wider well for light holes. The increase in effective well-width overcomes the effects of smaller mass and leads to a smaller quantization energy than for heavy holes, which still sees two coupled triangle wells with more or less the same width. In short, we see that doping induced band bending leads to a transition from a type-II to type-I heterostructure.

To study the effects of doping on interband transition matrix elements, we plot dipole matrix elements between the first electron subband and the light hole subband in Fig.2. Without doping, the dipole matrix elements are almost zero for low e-h density. We see that the dipoles increase with doping density. This is because of the increased spatial overlap between electrons and holes as the structure becomes more type-I like with increasing doping density. In addition, we see that, at relatively low doping density, the dipole also increases with e-h density. This is because both electrons and holes are attracted to the interface regions, an effect that is responsible for interband gain in other type-II structures [11]. This is a smaller effect however, compared to the dipole increase due to doping. The TM dipole changes only by about $0.5 \mathrm{~nm}$ within the e-h density change of $3 \times 10^{12}$, while it changes by about $1 \mathrm{~nm}$ with the change of doping density in the same range. At high doping level, the structure becomes already type-I. Further increase of e-h density does not help to increase the overlap any more. This is why, at the high doping level, all the curves for different e-h densities differ by little. In other words, the dipole moments become less sensitive to e-h density when the doping density is high. Another important point is that the TM dipole is 3-4 times larger than the TE dipole. This can be understood by comparing the bandedge profiles and wave functions for heavy and light hole bands.

We also calculated optical gain for the TE and TM modes using standard gain expression[8]. We use a Gaussian lineshape with the homogeneous linewidth of $10 \mathrm{meV}$. This linewidth value is in the upper range of the usually used value. The gain calculated in the following will be even larger if smaller linewidth is used. Examples of calculated spectrum are shown in Fig.3, where the e-h density is fixed, while TM and TE spectra are shown for various doping levels. As seen clearly, both peak gain magnitude and gain peak frequency change with doping level. The significant increase of gain magnitude is due to the 
increasing overlap of the electron and hole wavefunctions and increased electrons and holes in the InAs layer. We note that there is also an increase of the bandedges (lower energy edges of gain spectra) with doping density. This is mainly because the increased band bending leads to an energy increase of the ground state in the conduction band. At the same time, the transparency energy increases due to the increase of electrons with doping.

Finally peak gain is shown in Fig.4 for both TE and TM polarizations as a function of e-h density for various given doping densities. There are a few features worth noticing here: First, the TM gain is about 8 times larger than the TE gain. This is again mainly due to the large wavefunction overlap and the type-I transitions between conduction and light hole bands, while the overlap between conduction band and heavy hole band remains weak. Second, the magnitude of the TM mode gain is quite large (maximum $40001 / \mathrm{cm}$ !) for this wavelength region. Such magnitude is comparable to typical gain values seen for near infrared range, such as in InGaAs QWs. The TE gain is comparable to that of interband cascade lasers [12]. The third feature of the peak gain curves is the slower increase at the lower density region and the apparent absence of saturation for higher doping levels within the e-h density range we studied. This should lead to a less sensitive dependence of differential gain on e-h density than in standard type I and type II structures. In a typical type I structure without doping, the peak gain increases rapidly with density, reaches a saturation, and then starts to increase more gradually. This leads to a large differential gain at low density, but a dramatically reduced one for relatively high density. The lower panel of Fig. 4 shows the frequency of the gain peak as function of e-h density for both polarizations at various doping levels. We see that the peak frequency can be tuned by the doping level in the range of $340-390 \mathrm{meV}$ (3.6-3.2 $\mu \mathrm{m}$ in wavelength).

In summary, we demonstrated that proper doping can convert a well-known type II QWs such as InAs/AlSb into a type I structure, thus transforming an interband-inactive material system into a highly efficient interband gain structure. The TM gain is almost 10 times as large as in other MWIR gain material systems. We believe that this leads to an alternative gain structure in the technologically important MWIR wavelength range. Finally, this approach could be used to create gain material in other wavelength regions where lasing structures are still in great demand. An extension of this approach to other material systems and wavelength ranges will be discussed in a separate publication. 


\section{Acknowledgments}

This work is supported by a joint NASA-NCI program.

[1] B. Brar, H. Kroemer, J.Ibbetson, and J. English, Appl. Phys. Lett. 62, 3303 (1993).

[2] M. Inoue, M. Yano, H. Furuse, N. Nasu, and Y. Iwai, Semicond. Sci. Technol. 8, S121 (1993).

[3] F. Fuchs, J. Schmitz, J. Ralston, and P. Koidl, Phys. Rev. B 49, 13638 (1994).

[4] B. R. Bennett, B. V. Shannabrook, and E. R. Glaser, Appl. Phys. Lett. 65, 598 (1994).

[5] C. Becker, I. Prevot, X. Marcadet, B. Vinter, and C. Sirtori, Appl. Phys. Lett. 78, 1029 (2001).

[6] K.Ohtani, N. Matsumoto, H. Sakuma, and H. Ohno, Appl. Phys. Lett. 82, 37 (2003).

[7] J. Li et al., in Progress in Semiconductors II: Electronic and Optoelectronic Applications, edited by B. D. Weaver et al. (Materials Research Society, 2003).

[8] S. L. Chuang, Physics of Optoelectronic Devices (Wiley, New York, 1995).

[9] K. I. Kolokolov, J. Li, and C. Z. Ning, Phys. Rev. B (2003), to be published.

[10] I. Vurgaftman, J. R. Meyer, and L. R. Ram-Mohan, J. Appl. Phys. 89, 5815 (2001).

[11] G. Liu, S. L. Chuang, and S. Park, J. Appl. Phys. 88, 5554 (2000).

[12] G. Liu and S. L. Chuang, Phys. Rev. B 65, 1652201 (2002). 
Figure captions:

Fig.1 Bulk bandedge profiles (thick lines) and wave functions (thinner lines of various types) calculated for doping density $0.5 \times 10^{12} \mathrm{~cm}^{-2}$ (a) and $3 \times 10^{12} \mathrm{~cm}^{-2}$ (b) with the same e-h density $1 \times 10^{12} \mathrm{~cm}^{-2}$. The light and heavy hole band profiles are indicated by solid and dashed thick lines, respectively. Legends for wave functions are: first electron subband (solid), ground state (dashed) and first excited hole subbands (dot-dashed). Horizontal lines with the corresponding line styles indicate the subband energy levels.

Fig.2 TE and TM dipole matrix elements between the first electron subband and the light hole subband as a function of doping density for various e-h density levels indicated in the figure.

Fig.3 Gain spectra for the e-h density of $1.0 \times 10^{12}$ and for doping densities of $1.0,2.0, \ldots$ $5.0 \times 10^{12} \mathrm{~cm}^{-2}$ (in the order of increasing magnitude of gain), respectively. Note the different scales for the two panels.

Fig.4 The peak gain (upper panel) and peak frequency (lower panel) for TE (dashed curves) and TM (solid curves) modes as functions of e-h density for various given doping levels (the same as in Fig.3). Doping density for each curve increases from bottom to top. 


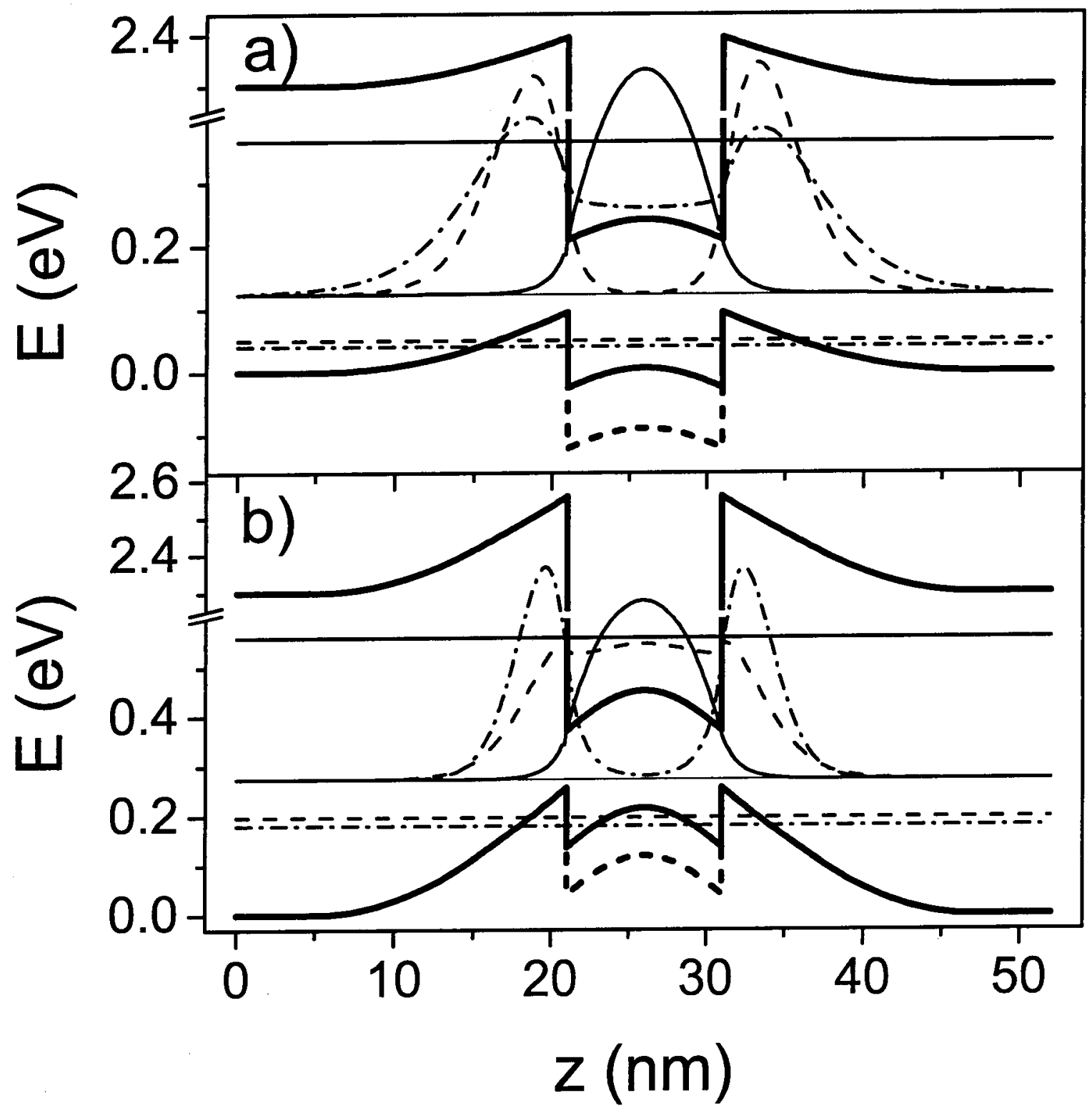

FIG. 1: Bulk bandedge profiles (thick lines) and wave functions (thinner lines of various types) calculated for doping density $0.5 \times 10^{12} \mathrm{~cm}^{-2}$ (a) and $3 \times 10^{12} \mathrm{~cm}^{-2}$ (b) with the same e-h density $1 \times 10^{12} \mathrm{~cm}^{-2}$. The light and heavy hole band profiles are indicated by solid and dashed thick lines, respectively. Legends for wave functions are: first electron subband (solid), ground state (dashed) and first excited hole subbands (dot-dashed). Horizontal lines with the corresponding line styles indicate the subband energy levels. 


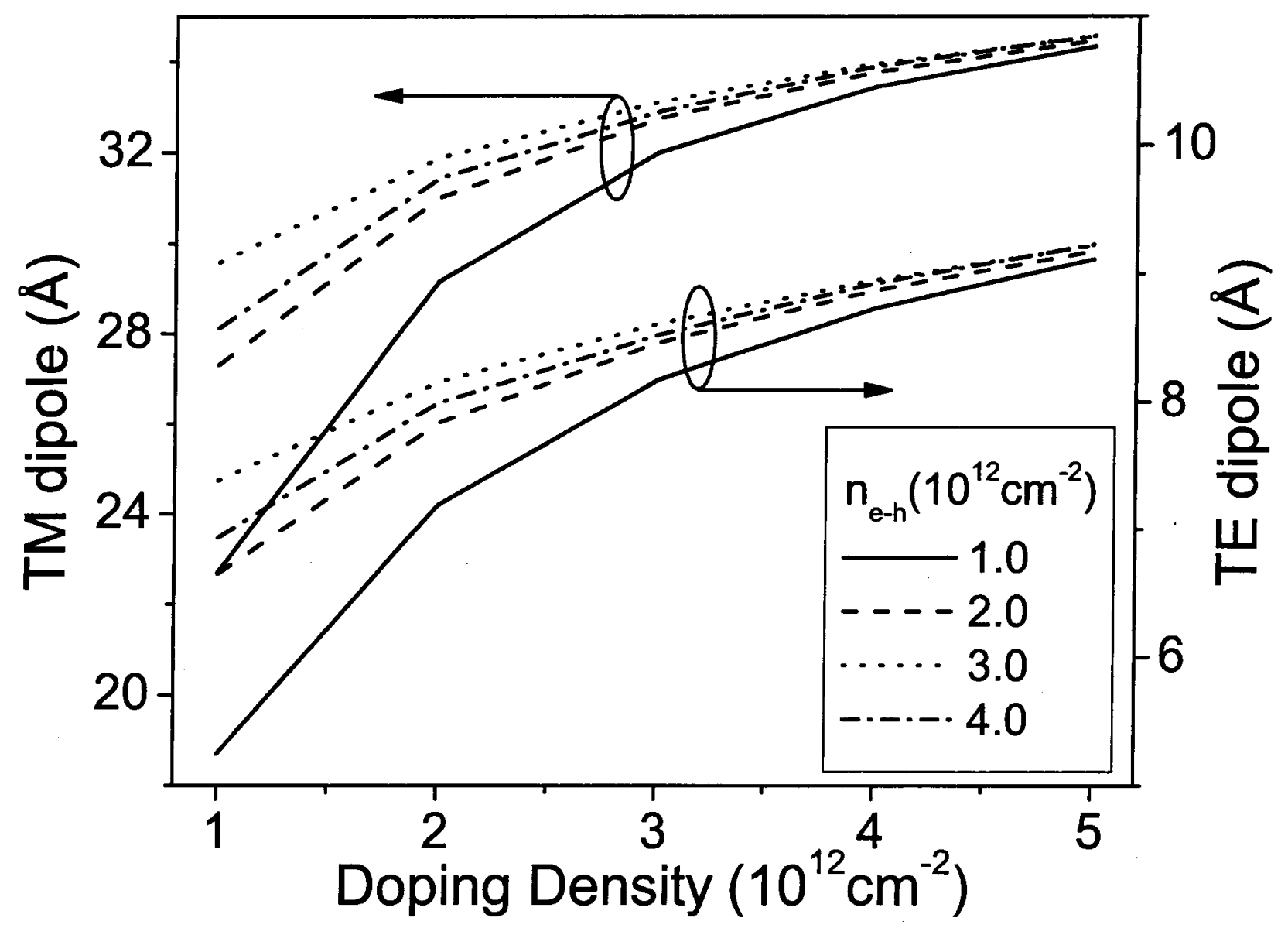

FIG. 2: TE and TM dipole matrix elements between the first electron subband and the light hole subband as a function of doping density for various e-h density levels indicated in the figure. 


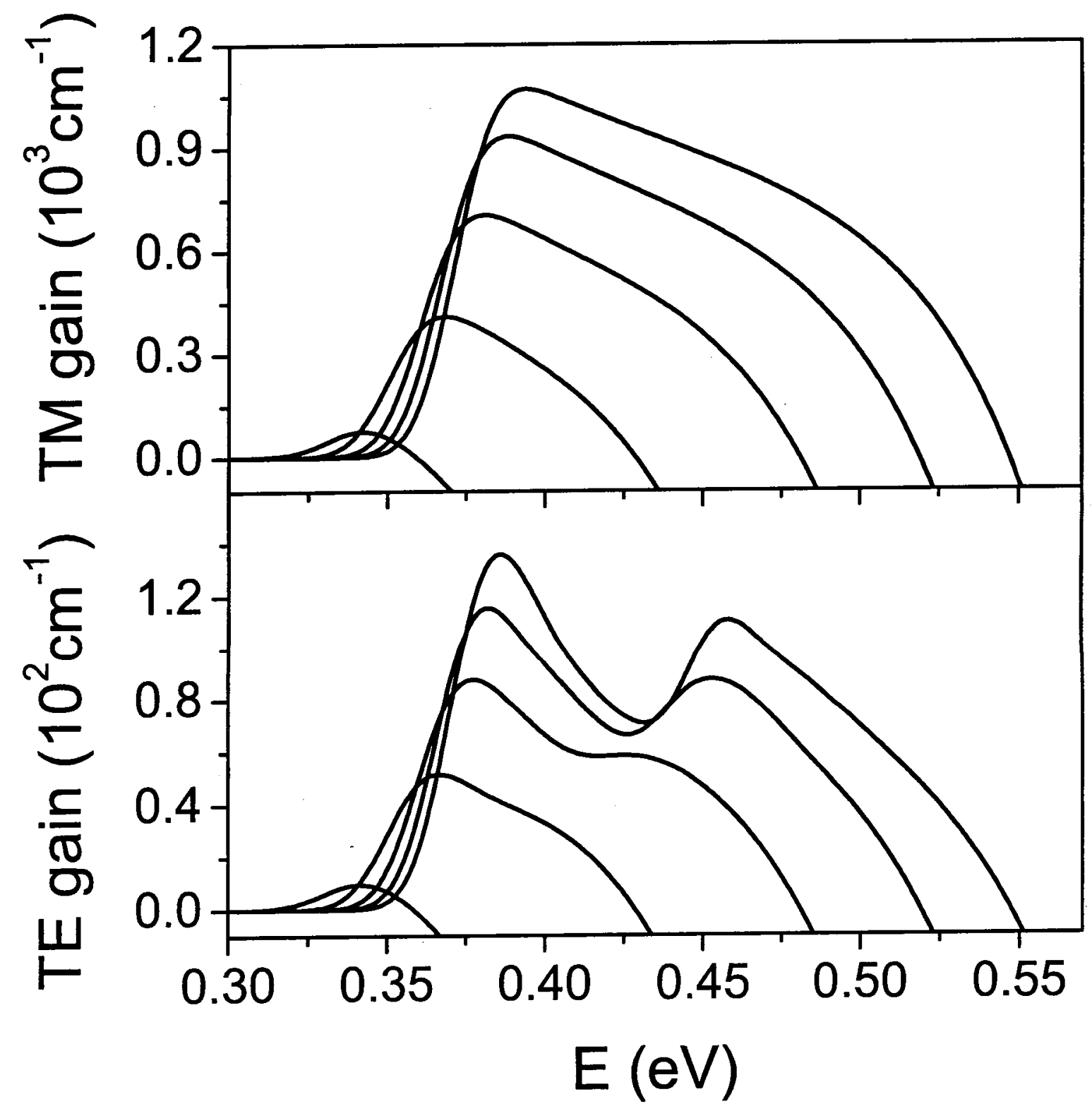

FIG. 3: Gain spectra for the e-h density of $1.0 \times 10^{12}$ and for doping densities of $1.0,2.0, \ldots$ $5.0 \times 10^{12} \mathrm{~cm}^{-2}$ (in the order of increasing magnitude of gain), respectively. Note the different scales for the two panels. 


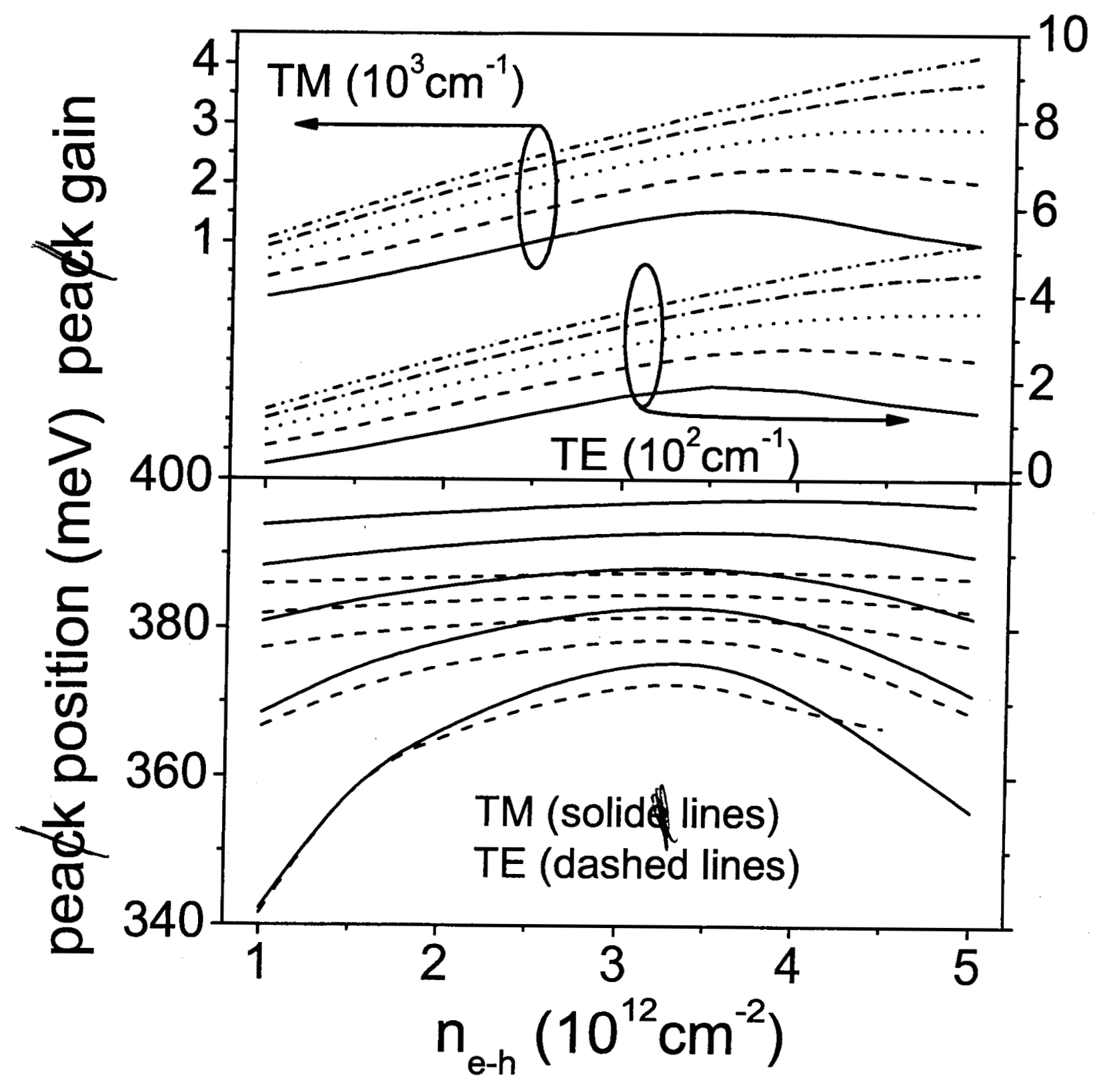

FIG. 4: The peak gain (upper panel) and peak frequency (lower panel) for TE (dashed curves) and TM (solid curves) modes as functions of e-h density for various given doping levels (the same as in Fig.3). Doping density for each curve increases from bottom to top. 